\title{
Emergent Structure in QCD
}

\author{
James Biddle ${ }^{1, *}$, Waseem Kamleh $^{1, * *}$, and Derek Leinweber ${ }^{1, * * *}$ \\ ${ }^{1}$ Centre for the Subatomic Structure of Matter, Department of Physics, \\ The University of Adelaide, SA 5005, Australia
}

\begin{abstract}
The structure of the $S U(3)$ gauge-field vacuum is explored through visualisations of centre vortices and topological charge density. Stereoscopic visualisations highlight interesting features of the vortex vacuum, especially the frequency with which singular points appear and the important connection between branching points and topological charge. This work demonstrates how visualisations of the QCD ground-state fields can reveal new perspectives of centre-vortex structure.
\end{abstract}

\section{Introduction}

Quantum Chromodynamics (QCD) is the fundamental relativistic quantum field theory underpinning the strong interactions of nature. The gluons of QCD carry colour charge and self-couple. This self-coupling makes the empty vacuum unstable to the formation of nontrivial quark and gluon condensates. These non-trivial ground-state "QCD-vacuum" field configurations form the foundation of matter.

There are eight chromo-electric and eight chromo-magnetic fields composing the QCD vacuum. An stereoscopic illustration of one of these chromo-magnetic fields is provided in Fig. 1. Animations of the fields are also available [1-3].

The essential, fundamentally-important, nonperturbative features of the QCD vacuum fields are the dynamical generation of mass through chiral symmetry breaking, and the confinement of quarks. But what is the fundamental mechanism of QCD that underpins these phenomena? What aspect of the QCD vacuum causes quarks to be confined? Which aspect is responsible for dynamical mass generation? Do the underlying mechanisms share a common origin?

One of the most promising candidates is the centre vortex perspective $[4,5]$. This perspective describes the nature of the QCD vacuum in terms of the most fundamental centre of the $S U(3)$ gauge group, characterised by the three values of $\sqrt[3]{1}$. Centre vortices on the lattice have been demonstrated to give rise to indicators of confinement such as the linear static quark potential [6-10] and enhancement of the infrared gluon propagator [11-14]. They also reproduce indicators of dynamical chiral symmetry breaking through enhancement of the infrared quark mass function $[15,16]$ and mass splitting in the low-lying hadron spectrum [16-18].

\footnotetext{
*e-mail: james.biddle@adelaide.edu.au

**e-mail: waseem.kamleh@adelaide.edu.au

***e-mail: derek.leinweber@adelaide.edu.au
} 


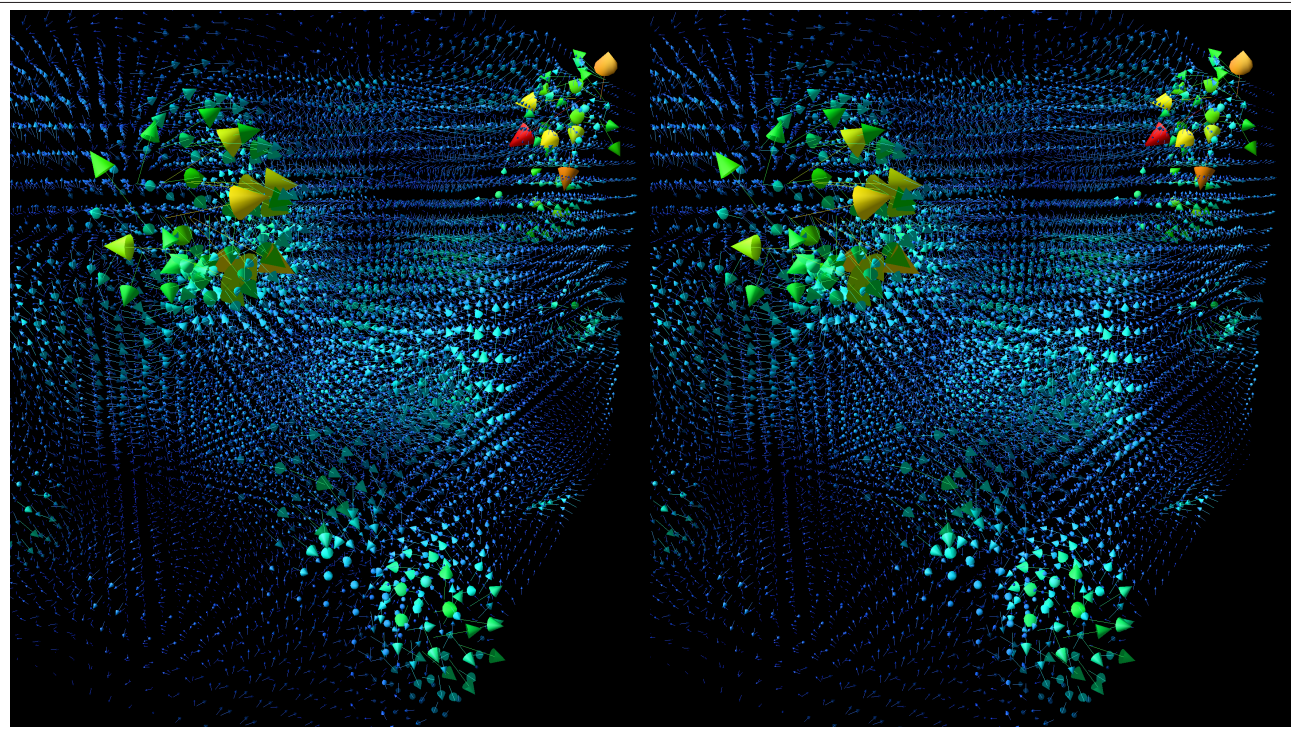

Figure 1. Stereoscopic image of one of the eight chromo-magnetic fields composing the nontrivial vacuum of QCD. Hints for stereoscopic viewing are provided in the text.

As such, it is interesting to ask, what do these structures look like? To this end, we present visualisations of centre vortices and topological charge density as identified on lattice gaugefield configurations. These visualisations are presented as stereoscopic images. To see the $3 \mathrm{D}$ image, try the following:

1. If you are viewing the image on a monitor, ensure the image width is 12 to $13 \mathrm{~cm}$.

2. Bring your eyes very close to one of the image pairs.

3. Close your eyes and relax.

4. Open your eyes and allow the (blurry) images to line up. Tilting your head from side to side will move the images vertically.

5. Move back slowly until your eyes are able to focus. There's no need to cross your eyes!

This work also features Josh Charvetto's augmented reality app for lattice QCD. Get the app using the QR code in Fig. 2 and see a whole new view of these visualisations through the camera's eye.

\section{Finding Centre Vortices}

Centre vortices are identified through a gauge fixing procedure designed to bring the lattice link variables as close as possible to the identity matrix multiplied by a phase equal to one of the three cube-roots of one $[7,19,20]$.

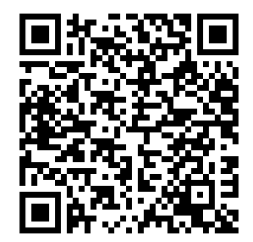

Figure 2. QR code to download the augmented reality app. 
The links $U_{\mu}(x)$ are then projected to the centre elements of $S U(3)$,

$$
U_{\mu}(x) \rightarrow Z_{\mu}(x), \quad \text { where } Z_{\mu}(x)=e^{2 \pi i m_{\mu}(x) / 3} \mathbf{I}, \quad \text { and } m_{\mu}(x)=-1,0,1,
$$

such that the gluon field is characterised by the most fundamental aspect of the $S U(3)$ link variable, the centre. This "vortex-only" field, $Z_{\mu}(x)$, can be examined to learn the extent to which centre vortices alone capture the essence of nonperturbative QCD.

Vortices are identified by the centre charge, $z$, given by the product of the vortex-only field around an elementary square or plaquette on the lattice

$$
z=\frac{1}{3} \operatorname{Tr} \prod_{\square} Z_{\mu}(x)=e^{2 \pi i m / 3} .
$$

If $z \neq 1$, a vortex with charge $z$ characterised by $m$ pierces the plaquette.

\section{Centre Vortex Features}

Vortex Lines: The plaquettes with nontrivial centre charge are plotted as red or blue jets piercing the centre of the plaquette in Figure 3. Here we are considering a 3D slice of the 4D space-time lattice at fixed time. The orientation and colour of the jets reflect the value of the nontrivial centre charge. Using a right-hand rule for the direction, plaquettes with $m=+1$ are illustrated by blue jets in the forward direction, and plaquettes with $m=-1$ are illustrated by red jets in the backward direction. Thus, the jets show the directed flow of centre charge $z=e^{2 \pi i / 3}$ through spatial plaquettes. They are analogous to the line running down the centre of a vortex as illustrated in Fig. 4.

Figure 3 exhibits rich emergent structure in the

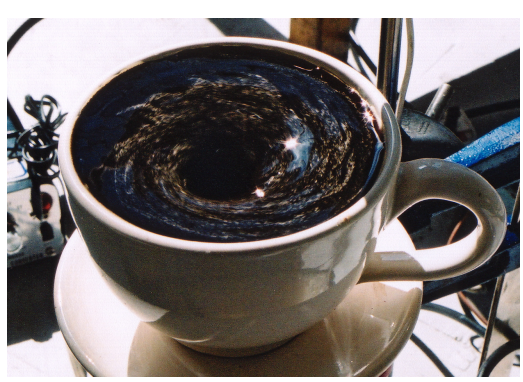

Figure 4. An example of a familiar vortex. The position of the vortex is characterised by the line running along the centre of the rotation. nonperturbative QCD ground-state fields.

Branching Points or Monopoles: In $S U(3)$ gauge theory, three vortex lines can merge into or emerge from a single point. Their prevalence is surprising, as is their correlation with topological charge density.

Vortex Sheet Indicator Links: As the vortex line moves in time, it creates a vortex sheet in 4D spacetime. This movement is illustrated by arrows along the links of the lattice (shown as cyan and orange arrows in Fig. 3 indicating centre charge flowing through the suppressed time direction.

Singular Points: When the vortex sheet spans all four space-time dimensions, it can generate topological charge. Lattice sites with this property are called singular points and are illustrated by spheres. The sphere colour indicates the number of times the sheet adjacent to a point can generate a topological charge contribution [21].

Topological Charge Density: Non-trivial topological charge is often associated with instanton-like field configurations which dynamically generate the mass the proton and other 


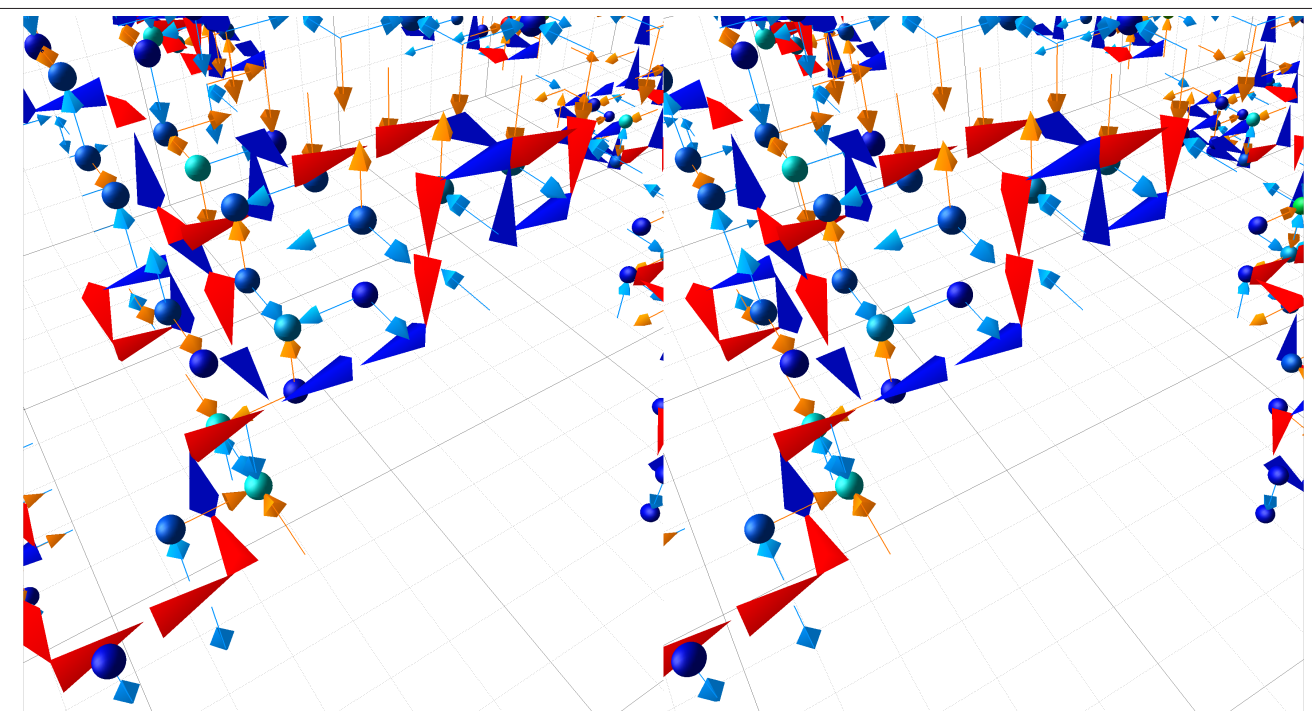

Figure 3. Stereoscopic image of centre vortices as identified on the lattice. Vortex features including vortex lines (jets), branching points (3-jet combinations), crossing points (4 jets), indicator links (arrows) and singular points (spheres) are described in the text.

hadrons. Topological charge density is plotted as red through yellow volumes for positive charge density and blue through green volumes for negative charge density in Fig. 5.

Vortices \& Topological Charge: Vortices are somewhat correlated with the positions of significant topological charge density, but not in a strong manner. However, the percolation of vortex structure is significant and the removal of these vortices destroys most instanton-like objects.

\section{Conclusion}

Visualisations of centre-vortex phenomena provide a fascinating glimpse into the structure of the QCD ground-state fields. Patterns and correlations that remain hidden within gigabytes of data become clear as one examines the data drawing on our highly-evolved sense of vision. It provides an extremely powerful form of data analysis. Animations of centre-vortex structure are also available [1, 22, 23].

A more detailed presentation of this work providing access to the full 3D models is available in Ref. [21]. It is interesting to reflect on the discoveries made in Ref. [21] through visualisation. These include:

- The high density of centre vortices and the complexity of their structure.

- The long-distance structure of the centre vortices as they percolate through the vacuum fields.

- The proliferation of branching points in $S U(3)$ gauge theory.

- The correlation of branching points with topological-charge-density peaks, revealing the vital role of vortex branching in generating topological charge density. 


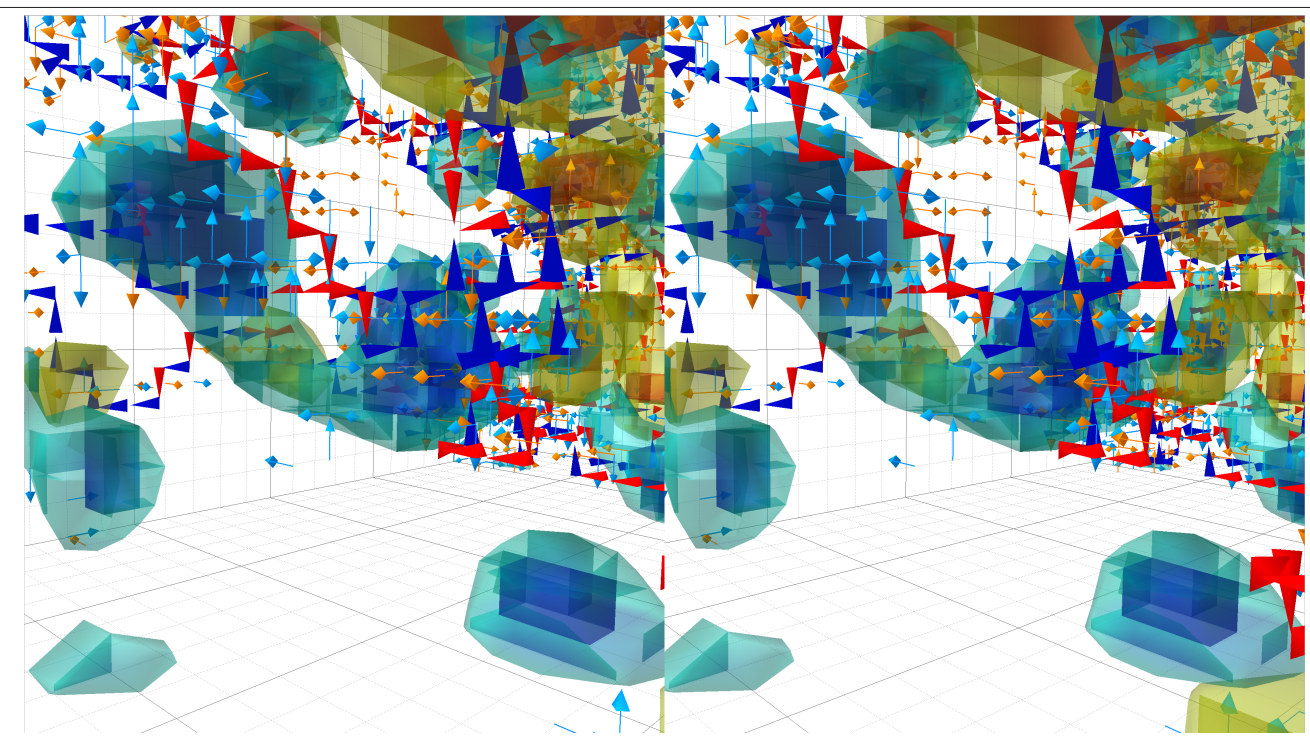

Figure 5. Stereoscopic image illustrating the correlation of topological charge density in the gluon field with the positions of centre vortices. Positive charge density is rendered red through yellow and negative charge density is rendered blue through blue.

- Understanding the sheet-like structure of centre vortices in four dimensions as they pierce one 3D time-slice of the lattice and traverse to the next.

- The speed with which centre vortices move through the lattice.

- The rapid loss of centre vortices under gauge-field smoothing routines.

The new understanding of centre-vortex branching and topological charge is important. This mechanism governs the size of instanton-like field configurations which generate a density of near zero-modes in the Dirac operator, dynamically breaking chiral symmetry in the groundstate fields and generating the mass of visible matter.

\section{Acknowledgements}

We thank Daniel Trewartha for his contributions to the gauge ensembles underlying this investigation. This research is supported with supercomputing resources provided by the Phoenix HPC service at the University of Adelaide and the National Computational Infrastructure (NCI) supported by the Australian Government. This research is supported by the Australian Research Council through Grants No. DP190102215, DP150103164, DP190100297 and LE190100021.

\section{References}

[1] J. Biddle, J. Charvetto, W. Kamleh, D. Leinweber, H. Piercy, E. Puckridge, F. Stokes, et al., "Publicizing Lattice Field Theory through Visualization," PoS LATTICE2018 (2019) 325, arXiv: 1903.08308 [hep-lat].

[2] D. B. Leinweber and E. Puckridge, Structure of the QCD Vacuum, 2019. https://youtu.be/WZgZI5vymiM. 
[3] D. B. Leinweber and E. Puckridge, Structure of the QCD Vacuum, 2018. http://www.physics.adelaide.edu.au/cssm/lattice/QCDvacuum/ChromoElectroMagneticFields.mp4.

[4] G. 't Hooft, "On the Phase Transition Towards Permanent Quark Confinement," Nucl.Phys. B138 (1978) 1.

[5] G. 't Hooft, "A Property of Electric and Magnetic Flux in Nonabelian Gauge Theories," Nucl.Phys. B153 (1979) 141.

[6] A. O'Cais, W. Kamleh, K. Langfeld, B. Lasscock, D. Leinweber, P. Moran, A. Sternbeck, and L. von Smekal, "Preconditioning Maximal Center Gauge with Stout Link Smearing in SU(3)," Phys. Rev. D82 (2010) 114512, arXiv:0807.0264 [hep-lat].

[7] K. Langfeld, "Vortex structures in pure SU(3) lattice gauge theory," Phys. Rev. D69 (2004) 014503, arXiv:hep-lat/0307030 [hep-lat].

[8] D. Trewartha, W. Kamleh, and D. Leinweber, "Connection between center vortices and instantons through gauge-field smoothing," Phys. Rev. D92 no. 7, (2015) 074507, arXiv: 1509.05518 [hep-lat].

[9] J. Greensite, "The Confinement problem in lattice gauge theory," Prog. Part. Nucl. Phys. 51 (2003) 1, arXiv:hep-lat/0301023 [hep-lat].

[10] L. Del Debbio, M. Faber, J. Giedt, J. Greensite, and S. Olejnik, "Detection of center vortices in the lattice Yang-Mills vacuum," Phys. Rev. D58 (1998) 094501, arXiv: hep-lat/9801027 [hep-lat].

[11] P. O. Bowman, K. Langfeld, D. B. Leinweber, A. Sternbeck, L. von Smekal, and A. G. Williams, "Role of center vortices in chiral symmetry breaking in SU(3) gauge theory," Phys. Rev. D84 (2011) 034501, arXiv: 1010.4624 [hep-lat].

[12] J. C. Biddle, W. Kamleh, and D. B. Leinweber, "Gluon propagator on a center-vortex background," Phys. Rev. D98 no. 9, (2018) 094504, arXiv: 1806.04305 [hep-lat].

[13] K. Langfeld, H. Reinhardt, and J. Gattnar, "Gluon propagators and quark confinement," Nucl. Phys. B621 (2002) 131-156, arXiv:hep-ph/0107141 [hep-ph].

[14] M. Quandt, H. Reinhardt, and G. Burgio, "The role of center vortices in Gribov's confinement scenario," Phys. Rev. D81 (2010), arXiv: 1001.3699 [hep-lat].

[15] P. O. Bowman, K. Langfeld, D. B. Leinweber, A. O' Cais, A. Sternbeck, L. von Smekal, and A. G. Williams, "Center vortices and the quark propagator in SU(2) gauge theory," Phys. Rev. D78 (2008) 054509, arXiv: 0806.4219 [hep-lat].

[16] D. Trewartha, W. Kamleh, and D. Leinweber, "Evidence that centre vortices underpin dynamical chiral symmetry breaking in SU(3) gauge theory," Phys. Lett. B747 (2015) 373-377, arXiv: 1502.06753 [hep-lat].

[17] D. Trewartha, W. Kamleh, and D. Leinweber, "Centre vortex removal restores chiral symmetry," J. Phys. G44 no. 12, (2017) 125002, arXiv: 1708.06789 [hep-lat].

[18] E.-A. O'Malley, W. Kamleh, D. Leinweber, and P. Moran, "SU(3) centre vortices underpin confinement and dynamical chiral symmetry breaking," Phys. Rev. D86 (2012) 054503, arXiv: 1112.2490 [hep-lat].

[19] L. Del Debbio, M. Faber, J. Greensite, and S. Olejnik, "Center dominance and Z(2) vortices in SU(2) lattice gauge theory," Phys. Rev. D55 (1997) 2298-2306, hep-lat/9610005.

[20] K. Langfeld, H. Reinhardt, and O. Tennert, "Confinement and scaling of the vortex vacuum of SU(2) lattice gauge theory," Phys. Lett. B419 (1998) 317-321, arXiv: hep-lat/9710068.

[21] J. C. Biddle, W. Kamleh, and D. B. Leinweber, "Visualisation of Centre Vortex Structure," arXiv: 1912.09531 [hep-lat].

[22] J. Biddle, W. Kamleh, D. Leinweber, and H. Piercy, Centre Vortices in the Gluon Field of the QCD Vacuum, 2019. https://youtu.be/CDdmx989quA.

[23] J. Biddle, W. Kamleh, D. Leinweber, and H. Piercy, Centre Vortices in the Gluon Field of the QCD Vacuum, 2018.

http://www.physics.adelaide.edu.au/cssm/lattice/QCDvacuum/CentreVorticesTopQ.mp4. 